\title{
Optoelectronic Moiré Projector for REAL-TIME SHAPE AND DEFORMATION STUdies OF THE TyMPANiC MembRane
}

\author{
Joris J. J. Dirckx and Willem F. Decraemer \\ University of Antwerp, Laboratory of Biomedical Physics, Groenenborgerlaan 171, B-2020 Antwerp, \\ Belgium \\ (Paper JBO-113 received Oct. 5, 1996; revised manuscript received Jan. 1, 1997; accepted for publication Feb. 20, 1997. )
}

\begin{abstract}
Moiré topography is a valuable technique for studying the shape and deformation of delicate biological structures such as the tympanic membrane. A projection moire method is described that can produce both shape and deformation fringes in real time, at a rate of 25 interferograms per second. The apparatus is based on electronic subtraction of the projected grating line image of the object and a reference grating line image stored in digital memory. The image field and fringe plane distance can be adjusted to the object dimensions. Using a fringe plane distance of $82 \mu \mathrm{m}$, a precision of $5 \mu \mathrm{m}$ over a measuring depth of $0.4 \mathrm{~mm}$ is demonstrated by measurements on a spherical calibration object. The application to in vitro shape and deformation measurements of the gerbil tympanic membrane is demonstrated. $\odot 1997$ Society of Photo-Optical Instrumentation Engineers. [S1083-3668(97)00202-5]
\end{abstract}

Keywords moiré, real time, eardrum.

\section{INTRODUCTION}

Various optical measurement techniques have proven to be valuable tools in the study of vibration, shape, and deformation of delicate biological structures such as the tympanic membrane. Interferometric techniques allow one to obtain fringes of equal height, equal displacement amplitude, or equal vibration amplitude on a diffusely reflecting object without touching its surface. From these fringes full-field measurements of object shape, deformation, and vibration amplitude can be made.

Time-averaged holographic interferometry has been used to investigate the vibration patterns of the tympanic membrane in vitro both in cat ${ }^{1}$ and in human ${ }^{2}$ material. Electronic speckle pattern interferometry have made it possible to study the vibrations of the human tympanic membrane in vivo. ${ }^{3}$ An overview of the applications of holography and related techniques in otologic research was presented by von Bally. ${ }^{4}$ More recently, in vivo highaccuracy measurements were presented of amplitude and phase of the cat vibrating tympanic membrane, using a heterodyning interferometer. ${ }^{5}$ These techniques even allow one to follow the motion of points on the tympanic membrane in three dimensions. ${ }^{6}$ Laser Doppler interferometers have also proven to be valuable tools for measuring the motion of the vibrating eardrum, ${ }^{7}$ and with its recent commercialization in an easy-to-use computer-

Address all correspondence to Joris J. J. Dirckx. E-mail: jdirckx@ruca.ua.ac.be controlled apparatus, the technique is becoming a widespread research tool that is even being used in the clinic (e.g., Refs. 8 through 10). Moire topography is a powerful technique for measuring the shape of the eardrum, ${ }^{11}$ and investigating its deformations caused by static middle ear pressures. ${ }^{12,13}$

In research in otology, the understanding of the effect of middle ear pressure upon the eardrum is important both from a clinical point of view and for fundamental studies of middle ear mechanics. We have measured in vitro the deformation of the eardrum under static pressure in preparations of healthy human temporal bones, ${ }^{12}$ and in preparations of Mongolian gerbils with healthy ${ }^{13}$ and diseased ${ }^{14}$ middle ears. For the measurement of these (relatively large) deformations, we have found moire topography to be the technique of choice because no coherent light is needed and the sensitivity of the setup can be easily controlled by using different gratings and/or different setup geometries. The best results are obtained for surfaces with strong homogeneous diffuse reflections. Reflective characteristics may be improved, if necessary, by application of a coating such as white paint to the object surface.

In shadow moiré topography, a point light source casts the shadow of a transmission grating onto the surface of the object. $^{15,16}$ The setup for shadow moiré topography is essentially very simple, but has the major drawback that the object needs to be 


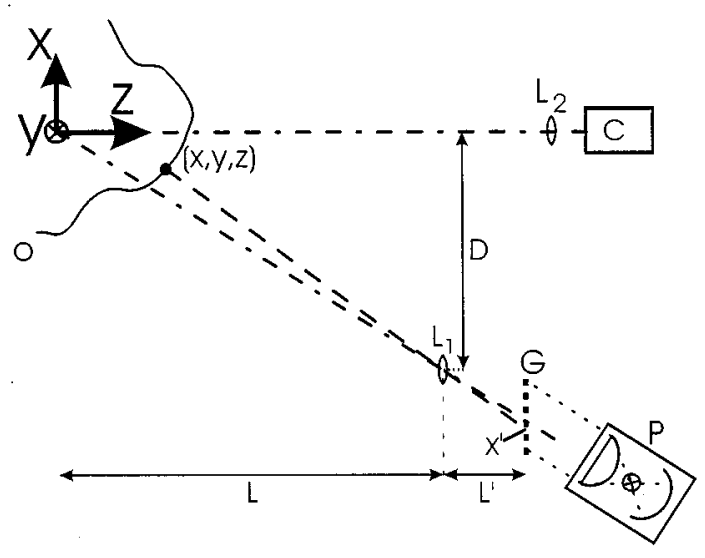

Fig. 1 Schematic diagram of the optical setup. The projector $(P)$ casts light on the transmission grid $(G)$. This grid is projected by a lens $\left(L_{1}\right)$ onto the surface of the object $(O)$. The grid lines, modulated by the object surface shape, are imaged onto the camera target $(C)$ by a second lens $\left(L_{2}\right)$. The distances $D, L$, and $L^{\prime}$ and the grid period determine the setup fringe plane distance.

positioned very close to the grating because the depth of field of the shadow is very limited. In projection moire topography, ${ }^{17,18}$ the image of a grating is projected onto the object surface from a distance. This technique requires the use of a more elaborate optical setup, but has the advantage that the object can be freely positioned in space. For research in otology, this is a major advantage, even for in vitro studies, because it is difficult to remove enough bone to get immediate access to the tympanic membrane.

In classical projection moiré topography, ${ }^{17,18}$ a transmission ruling is imaged on the surface of the object. The projected grid lines are modulated by the object's shape. This deformed ruling is then imaged by a second lens onto another identical ruling. The interference between the deformed and nondeformed grid lines produces moiré fringes, which can be interpreted as contours of equal object surface height. Deformation measurements have to be done by obtaining moire topograms of the object in an original and a deformed state, followed by reconstruction of the object shape from these topograms and finally calculating the difference between these two shape measurements.

In this paper we describe an optoelectronic projection moiré system that does not use a second ruling for demodulation, and that allows one to obtain in real time contours of equal height as well as contours of equal deformation. The apparatus was developed for in vitro shape and deformation studies of the tympanic membrane.

\section{THEORY OF FRINGE FORMATION}

Let us consider the projection setup represented schematically in Figure 1. The light of projector $P$ is passed through the grid $G$ and projected by lens $L_{1}$ onto the surface of the object. The projected grid lines are modulated by the object shape and are imaged by a second lens $L_{2}$ onto the camera $C$. The focal points of the projection and imaging system coincide at the origin of an orthogonal coordinate system. The $z$ axis of the coordinate system is chosen along the viewing direction, and the $x$ axis in the plane formed by the projection and imaging axes.

For a sinusoidal transmission ruling with grid lines parallel to the $y$ axis, the modulation function $T$ is given by:

$$
T=I_{0}\left[\frac{1}{2}+\frac{1}{2} \sin \left(\frac{2 \pi}{p} x^{\prime}\right)\right],
$$

where $I_{0}$ is the intensity of the incoming light (the projector), $p$ is the period of the grid, and $x^{\prime}$ is the $x$ axis coordinate of the point on the grid where the light emerges. The $y$ axis coordinate does not appear in the equation since the grid lines are positioned parallel to the $y$ axis so that for a given value of $x^{\prime}$ the modulation is identical for all values of $y$.

The light reaching a point on the surface of the object with coordinates $(x, y, z)$ is coming from a point on the grid with coordinates $\left(x^{\prime}, y^{\prime}\right)$. In Figure 1 we can define two similar triangles: one with orthogonal sides of length $x^{\prime}-x$ and $L+L^{\prime}-z$, and another with orthogonal sides of length $D-x$ and $L-z$. From these two similar triangles we see that the position $x^{\prime}$ on the grid is determined by:

$$
\frac{x^{\prime}-x}{L+L^{\prime}-z}=\frac{D-x}{L-z}
$$

or

$$
x^{\prime}=\frac{(D-x)\left(L+L^{\prime}-z\right)}{L-z}+x,
$$

where $D$ is the distance along the $x$ axis between the projection lens and the viewing direction, $L$ is the distance along the $z$ axis between the grid and $L_{1}$, and $L^{\prime}$ is the distance between the object and $L_{1}$.

When we substitute Eq. (3) into Eq. (1), we see that the camera will observe a light distribution $I$ on the object given by:

$$
I=I_{0}\left[\frac{1}{2}+\frac{1}{2} \sin \left\{\frac{2 \pi}{p}\left[\frac{(D-x)\left(L+L^{\prime}-z\right)}{L-z}+x\right]\right\}\right] .
$$

In an ordinary projection moiré setup, this light distribution is demodulated by multiplying it by the transmission function of a second ruling. In practice, this multiplication is obtained by passing the light through a second ruling positioned in the focal plane of $L_{2}$. In our setup, there is no second 
grid, and the light distribution given by Eq. (4) is projected directly by $L_{2}$ onto the camera sensor, without demodulation.

After recording the intensity distribution given by Eq. (4), a second intensity distribution is recorded for an object with a different shape (which may be the original object after it has undergone a deformation). On the point with coordinates $(x, y)$ this object has a height that we will denote as $z^{\prime}$, so the recorded intensity distribution will be once again given by Eq. (4), but with $z^{\prime}$ substituted for $z$.

To obtain the moire interference, these two intensity distributions are subtracted from one another, which yields the difference $I_{d}$ :

$$
\begin{aligned}
I_{d}= & \frac{I_{0}}{2}\left[\sin \left\{\frac{2 \pi}{p}\left[\frac{(D-x)\left(L+L^{\prime}-z\right)}{L-z}+x\right]\right\}\right. \\
& \left.-\sin \left\{\frac{2 \pi}{p}\left[\frac{(D-x)\left(L+L^{\prime}-z^{\prime}\right)}{L-z^{\prime}}+x\right]\right\}\right]
\end{aligned}
$$

or:

$$
\begin{aligned}
I_{d}= & I_{0} \cos \left\{\frac { 2 \pi } { p } \left[\frac{(D-x)\left(L+L^{\prime}-z\right)}{2(L-z)}\right.\right. \\
& \left.\left.+\frac{(D-x)\left(L+L^{\prime}-z^{\prime}\right)}{2\left(L-z^{\prime}\right)}+x\right]\right\} \\
& \times \sin \left\{\frac { 2 \pi } { p } \left[\frac{(D-x)\left(L+L^{\prime}-z\right)}{2(L-z)}\right.\right. \\
& \left.\left.-\frac{(D-x)\left(L+L^{\prime}-z^{\prime}\right)}{2\left(L-z^{\prime}\right)}\right]\right\} .
\end{aligned}
$$

If we take the setup dimensions $D$ and $L$ as being large compared with the values of $x$ and $z$ for all points on the object, Eq. (6) simplifies to:

$$
I_{d}=I_{0} \cos \left\{\frac{2 \pi}{p} x+\varphi\right\} \sin \left[\frac{2 \pi}{p} \frac{D\left(z^{\prime}-z\right)}{2 L}\right],
$$

where

$$
\varphi=\frac{2 \pi}{p}\left[\frac{D\left(L+L^{\prime}\right)}{L}+\frac{D\left(z-z^{\prime}\right)}{2 L}\right] .
$$

Equation (7) contains the product of a cosine factor, with the same frequency as the projected ruling, and a sine factor, which is only dependent on the difference in surface height $\left(z^{\prime}-z\right)$ between the two objects. In the image, the cosine factor is seen as high spatial frequency grid lines, referred to as grid noise. The sine factor can be regarded as an amplitude modulation of the cosine factor and it describes contours of equal height displacement. The term $\varphi$ in the cosine argument can be regarded as a phase offset, although it is not constant over the entire image: the first term in Eq. (8) is constant over the whole image, and accounts for the shift of the grid lines as a whole when the setup geometry is altered, but the second term in (8) shows that $\varphi$ is also dependent on the difference $z-z^{\prime}$ which in turn depends on $x$. The phase $\varphi$ therefore varies slightly over the image. One could also interpret this varying phase as a (slight) spatial modulation of the cosine frequency. Because $L$ is much larger than $D$, the effect of changing $z-z^{\prime}$ on the cosine frequency is small.

\subsection{SHAPE MEASUREMENT}

If we use as a second object a flat plate positioned in the origin and perpendicular to the viewing direction, so that $z^{\prime}$ equals 0 for all values of $(x, y)$, Eq. (7) reduces to:

$$
I_{s}=I_{0} \cos \left[\frac{2 \pi}{p}(x+\varphi)\right] \sin \left(\frac{2 \pi}{p} \frac{D z}{2 L}\right) .
$$

In Eq. (9), the sine factor describes contours of equal surface height, so from $I_{s}$ we can determine the shape of the object.

\subsection{DEFORMATION MEASUREMENT}

If we do not replace the object by a flat plate, but leave it in place and make it undergo some kind of deformation, then Eq. (7) describes fringes of equal displacement along the $z$ axis, so $I_{d}$ can be used to measure the deformation of the object along the $z$ axis.

\subsection{FRINGE CHARACTERISTICS}

The fringes described by Eqs. (7) and (9) are not dark and bright fringes as we are used to seeing in topography. In zones where the sine argument is 0 , the observed intensity becomes 0 (seen as black contours), but elsewhere high spatial frequency dark and bright grid lines (grid noise) will be seen. The height or displacement difference between two subsequent black contours is determined by the argument of the sine factor, and is thus given by:

$$
\lambda=\frac{p L}{D} .
$$

The distance $\lambda$ is called the fringe plane distance, because in shape measurements the fringes can be thought of as the intersection of the object with a set of equidistant planes separated by the distance $\lambda$ along the $z$ axis.

Because of the term $D\left(z^{\prime}-z\right) / L$ in the cosine argument of Eq. (8), the lines in the striped contours do not have exactly the same spatial frequency as the original grid. The exact frequency of the stripes seen in the bright contours is, however, irrelevant to the interpretation of the moire topogram.

\subsection{FRINGE ENHANCEMENT}

In practice, the two intensity distributions of the two different objects may not have the same amplitude, owing to differences in object reflectivity. If 
this is the case, the difference in intensity will not vary around 0 , but around some offset value. This offset can be removed by applying a high-pass filter to the subtracted image, which only passes the high-frequency cosine function, but not a dc offset.

Negative intensities can of course not be visualized, so half of the cosine signal in Eq. (7) is lost. Only the positive parts of the cosine are seen as light, whereas the negative parts are seen as 0 intensity. The mean amplitude in the brightest zone of the fringe (sine argument equal to 1 or -1 ) therefore equals the rms value of a half-wave rectified cosine, namely 0.35 .

The signal could be made all positive by adding an offset (as will be done in the image presented in Figure 4 in the results section), but then the zones where the sine is 0 would be seen as gray, and the zones where the sine becomes maximum would be seen as high-frequency grid lines but with the same mean intensity as the gray zones. Fringes are then visualized as zones of high and low grid line contrast, and will be difficult to see if very fine rulings are used.

Instead of adding an offset, we can also make the signal all positive by taking its absolute value, which can be done in practice by performing a fullwave rectification of the video signal. Mathematically, the difference intensity distribution $I_{d}^{\prime}$ can be written as:

$$
I_{d}^{\prime}=I_{0}\left\{\cos ^{2}\left[\frac{2 \pi}{p}(x+\varphi)\right] \sin ^{2}\left[\frac{2 \pi}{p} \frac{D\left(z^{\prime}-z\right)}{2 L}\right]\right\}^{1 / 2},
$$

or:

$$
\begin{aligned}
I_{d}^{\prime}= & I_{0}\left[\left\{\frac{1}{2}+\frac{1}{2} \cos \left[\frac{4 \pi}{p}(x+\varphi)\right]\right\}\right. \\
& \left.\times\left\{\frac{1}{2}+\frac{1}{2} \sin \left[\frac{2 \pi}{p} \frac{D\left(z^{\prime}-z\right)}{L}\right]\right\}\right]^{1 / 2} .
\end{aligned}
$$

The second factor of the square root in Eq. (12) once again describes a slow modulation, which depends on the surface height. The first factor describes the grid noise, which now varies between 0 and 1 . In zones where the sine equals -1 , the observed intensity is 0 . In zones where the sine becomes equal to 1 , the mean amplitude now equals that of a fullwave rectified cosine, namely 0.7 , so the contrast of the fringes is doubled compared with Eq. (7). Moreover, Eq. (12) shows that the spatial frequency of the grid noise seen in a bright fringe now equals $2 / p$, which is double the spatial frequency of the ruling. This frequency doubling due to the rectification process makes the grid noise less disturbing and further enhances fringe visibility.

The derivation given above only holds for the case of an ideal setup. A rigorous mathematical analysis of a practical setup is far more complex, and falls beyond the scope of the present paper. A practical setup uses for instance square wave grids instead of sinusoidal grids, so the exact derivation should take the higher harmonics into account. Owing to its limited field angle, the projection lens has to be positioned at right angles to the projection direction rather than parallel to the imaging plane, which will cause a slight change in magnification of the grid lines over the image field, and this will result in a slight curvature of the fringe planes. The use of finite apertures limits the depth of field of both the projection and the imaging system, so that in practice fringe contrast diminishes with object depth.

The use of square wave grids instead of sinusoidal grids can be incorporated in the mathematical treatment by using the Fourier series which describes the square grid as a sum of sine grids. The outcome of the derivation is essentially the same as for the single sine grating, but all equations become much more lengthy. The incorporation into the mathematical description of the effects of finite apertures and Keystone distortions is far more complex. Even this description is not fully accurate because, for instance, the transfer characteristics of the lens will affect the harmonic content of the projected grids and will therefore further (strongly) complicate a complete mathematical model of the fringe formation process. The outcome of a rigorous and complex mathematical treatment could help to calculate quantitative estimates of measuring accuracy and precision on theoretical grounds. The simplified derivation, however, suffices for understanding the fringe formation process. The actual measuring performance of the practical setup will be shown experimentally in the next sections. Such an experimental approach incorporates all physical characteristics and limitations of the setup and yields quantitative estimates of measuring precision for a real-life setup in an easy way.

\section{Apparatus}

\subsection{OPTICAL SETUP}

The optical setup is essentially the same as for any other projection moiré setup, and is schematically represented in Figure 1. The frame grabber, digital subtraction electronics, and signal processing electronics were all designed and built in our electronics workshop. The light of a 100-W halogen lamp projector is passed through a 300-lines per $25.4 \mathrm{~mm}$ transmission grid $(G)$ and is projected by a $100-\mathrm{mm}$ focal length photographic lens $\left(L_{1}\right)$ onto the surface of the object $(O)$. A second identical lens $\left(L_{2}\right)$ images the deformed grid lines onto a CCD camera (Sony AVE-D7CE) (C). In contrast to a normal projection moire setup, there is no second grid in the focal plane of the imaging lens: the projected grid lines are not optically demodulated, but are directly recorded by the camera. 
The projection and imaging optics are mounted on optical rails that can pivot in the $x-y$ plane around the focal point. In this way, the angle between projection and imaging direction, and hence the fringe plane distance or sensitivity of the setup, can be easily changed while the object is in place and remains in focus for both the imaging and the projection systems. The camera and imaging lens are mounted on a translation rail and the imaging lens is coupled to the camera with a bellows, so that the field of view of the CCD camera can be adjusted to the object size. In our experiments, the grid-toobject distance and the camera-to-object distance is on the order of $40 \mathrm{~cm}$, and the angle between projection and imaging direction is about $35 \mathrm{deg}$.

\subsection{ELECTRONIC IMAGE PROCESSING}

The image-processing electronics are represented schematically in Figure 2. The camera signal is digitized by an analog-to-digital converter $(A / D)$ with a resolution of 8 bits, at a rate of 25 images per second. One complete frame (two interlaced frames) of digitized video data is stored in a 512 by 512-pixel memory matrix. The memory of this frame store consists of $250 \mathrm{kB}$ of inexpensive standard computer dynamic random access memory. When the frame store is set to free run mode, the previous video frame is cleared and replaced by the next video frame coming from the camera. When the frame store is set to hold mode, the last incoming video frame is stored and remains in the memory until the frame store is again released.

The data in the frame store are continuously read at a speed of 25 image frames per second. These data go into the subtraction unit, which can be set to either direct or subtraction mode. In direct mode, the frame store data are simply passed on to the output of the unit. In subtraction mode, the digitized real-time image data coming from the camera are subtracted from the data coming from the frame store. This subtraction process is also performed in real time, at a rate of 25 images per second, using a commercially available subtraction chip (model 74181). The data at the output of the subtraction unit are converted back to an analog video signal by a digital-to-analog converter $(\mathrm{D} / \mathrm{A})$.

If the subtraction unit is set to direct mode, a monitor connected to point A displays the stored image when the frame store is set to hold. The same monitor can be used to look at the direct image by switching the frame store to free run. Monitoring the direct camera image is necessary when positioning and focusing an object in the setup. If an image is captured in the frame store, and the subtraction mode is chosen, the monitor at point $\mathrm{A}$ will show the image described by Eq. (7). We will show such an image in the results section.

The difference signal coming from the subtraction unit contains the difference between the reference image and direct camera image. The signal is fed

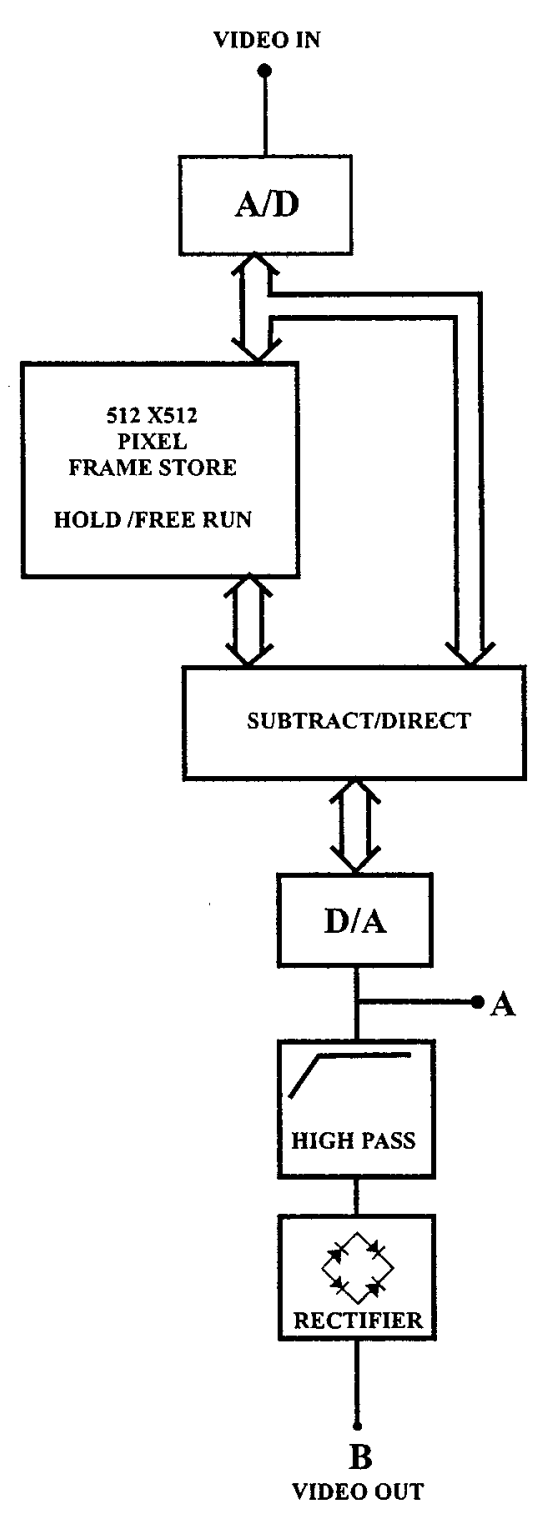

Fig. 2 Block diagram of the image-processing electronics. The incoming video signal is digitized by an 8-bit analog-to-digital converter. The digitized image can be stored in a 512 by 512 pixel frame store. In the subtraction unit, the difference is taken between the stored image and the incoming video signal, at a rate of 25 images per second. A digital-to-analog unit converts the difference data back to an analog video signal. High-pass filtering and fullwave rectification of the analog video signal allow real-time enhancement of fringe visibility, as explained in the text.

through a high-pass filter to remove offset, and fullwave rectified by a bridge rectifier to take the absolute value. Because the camera image is read in a horizontal direction, one line after the other, this processing of the video time signal is equivalent to filtering and rectifying the image data along the $x$ axis. By performing the high-pass filtering and rectification on the analog video signal, no complicated and fast digital signal processor is needed to obtain the processed image in real time. The moiré fringes described by Eq. (12) are finally displayed 
on a monitor connected to point $B$, at a rate of 25 new interferograms per second.

\section{MeAsurement ReSUlts}

\subsection{ADJUSTMENT AND CALIBRATION}

For studies on objects of different sizes, such as tympanic membranes of different species, we use different magnification factors. The field of view can be adjusted to the object size by using different lens-to-object distances. By using different rulings or a different projection-to-observation angle, the fringe plane distance can easily be adapted.

To accurately measure the shape or deformation of an object from the moiré fringe pattern, the fringe plane distance $\lambda$ has to be known precisely. Because it is impractical to pinpoint the distances $L$ and $D$ with high accuracy, the fringe plane distance is obtained by direct calibration, rather than by calculating it from the geometrical parameters. To perform the calibration measurement, an object is mounted on a calibrated translation table with the translation axis of the stage parallel to the viewing direction. The grid line image on the object is stored in the frame store, and the processed subtraction image is monitored. When the object is moved backward along the $z$ axis using the translation stage, the processed image turns alternately bright and dark each time as the displacement grows with one fringe plane distance. First, the object is positioned so that it turns completely dark. Then the object is translated over one or more fringe plane distances until it is again dark. The local minimum in the intensity is easily judged by eye on the monitor image. The localization of this minimum is repeatable within a few micrometers. The fringe plane distance is calculated from the translation distance, read from the micrometer screw on the translation stage, and divided by the number of fringe planes encountered in the translation. To obtain high accuracy, we perform the calibration by measuring the translation distance for ten fringe orders. As we explained in the previous section, the fringe plane distance varies slightly with object height. Calculating $\lambda$ from the distance of ten fringe planes gives an average fringe plane distance within this depth measuring range.

For the measurements presented in this section, the fringe plane distance was set at $0.082 \mathrm{~mm}$. This setting was also used for our studies on gerbil tympanic membranes. ${ }^{13,14}$ With projection and lens apertures of $f / 16$, the setup gives good fringe contrast over a depth range of about 25 fringe orders or 2 $\mathrm{mm}$. The field of view for the processed image was $3.5 \mathrm{~mm}$ horizontally and $2.9 \mathrm{~mm}$ vertically. This is somewhat smaller than the actual camera image, because the frame store does not capture the video image over its full width.

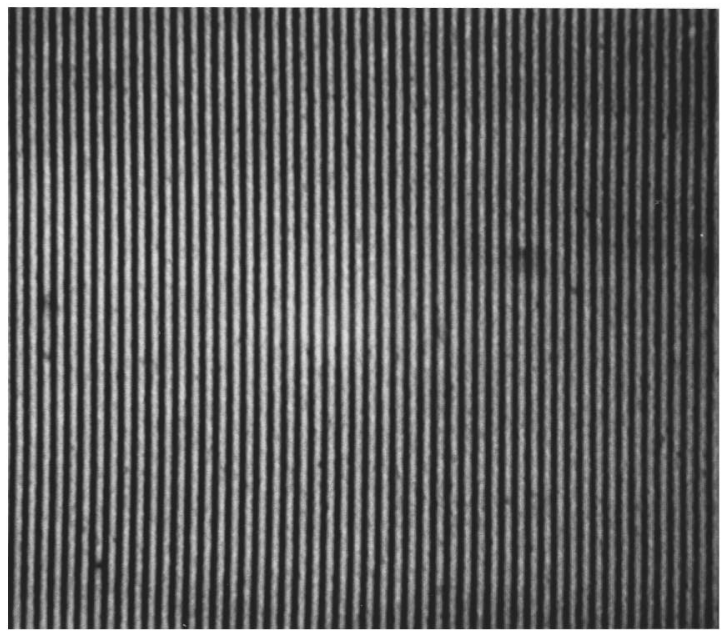

(a)

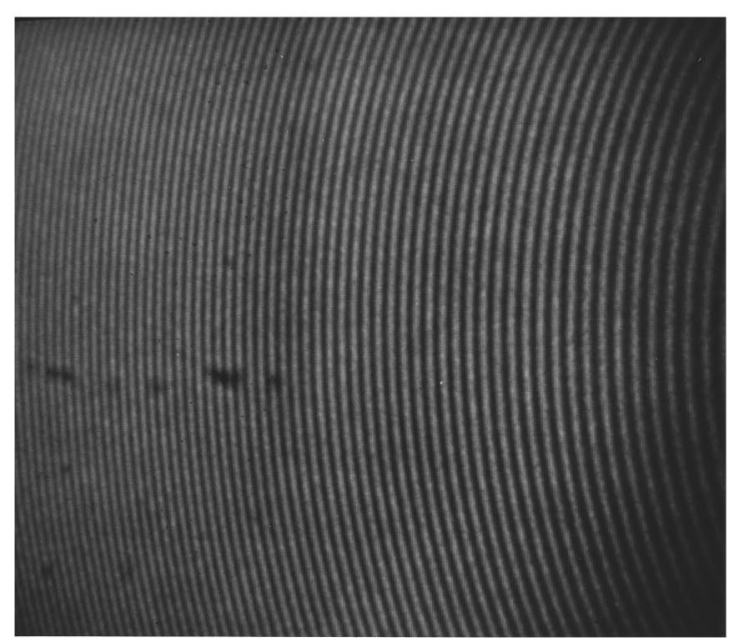

(b)

Fig. 3 (a) Direct image of the grid lines projected onto a flat surface positioned parallel to the $x-y$ plane. The projected lines are straight and equidistant. (b) Direct image of the grid lines projected onto the surface of a sphere. The grid lines bend due to the surface shape and the angle between the projection and the direction of observation.

\subsection{MEASURING RESOLUTION}

To demonstrate the measuring resolution, we show results obtained on a small sphere. The test object is a steel bearing ball with a spherical surface precision better than $1 \mu \mathrm{m}$. The diameter of the ball is $6.750 \pm 0.005 \mathrm{~mm}$. Because diffuse reflection is needed in projection moiré topography, the surface of the ball was coated with white Chinese ink. Scanning electron microscopy on samples of this coating has taught us that the layer thickness is uniform and less than $10 \mu \mathrm{m}$, so it will only cause very small artifacts on the surface shape.

Figure 3(a) shows the direct image of the grid projected onto a flat surface positioned in the $x-y$ plane: the projected grid lines are straight and equidistant. Figure 3(b) shows the direct image of the grid projected onto the surface of the sphere. We 


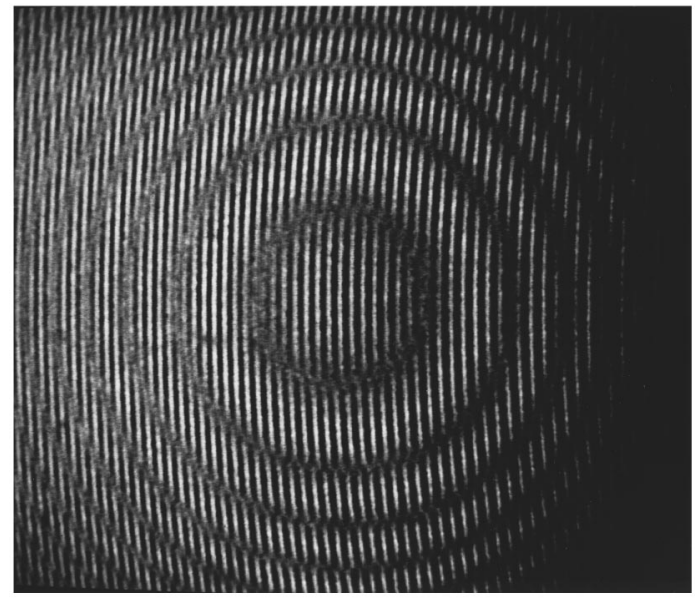

Fig. 4 Image displayed by a monitor connected to point $A$ of Figure 2. To obtain this image, the grid line image of Figure $3(b)$ was stored in the frame store and was subsequently subtracted from the image of the grid lines projected on a flat plate, shown in Figure $3(a)$. The gray and striped circular bands are contours of equal height of the sphere surface.

see the projected grid lines bending due to the spherical surface shape. At the right-hand side, the image is darker due to shadow effects caused by the strong curvature of the ball surface and the large angle between illumination and observation direction. The moiré fringes are generated by subtracting the two images in Figure 3. The image shown in Figure 3(b) is captured in the frame store and then the sphere is replaced by the flat plate positioned parallel to the $x-y$ plane. Figure 4 shows a photograph of the image displayed by a monitor connected to point A in Figure 2, when the setup is switched to subtraction mode. We recognize circular bands in this image which are alternating gray and striped. These bands are the fringes described by Eq. (9). The electronics of the display monitor automatically generate an offset so that the video signal becomes completely positive. In this way, the negative intensity minima of the grid stripes $\left[l_{s}=-l_{0}\right.$ in Eq. (9)] are put at 0 level (black) and as a consequence the dark fringes [sine factor in Eq. (9) equal to 0] become gray. Although it is perfectly possible to follow the fringes, their visibility is not very good, especially in the zones where the fringes are close to one another. Figure 5 shows the result after high-pass filtering and half-wave rectification of the signal, as displayed on a monitor connected to point $\mathrm{B}$ shown in the electronics scheme presented in Figure 2. In this image we have clear bright and dark fringes, described by Eq. (12). When we compare the grid lines in Figures 4 and 5, we see that their frequency has doubled in the last image, as predicted by Eq. (12). Because the fringes are obtained in real time, any motion of the sphere can be followed at a rate of 25 interferograms per second. At the right-hand side of the image, fringe quality is somewhat lower due to the weaker illumination in this zone caused by the shadow effect.

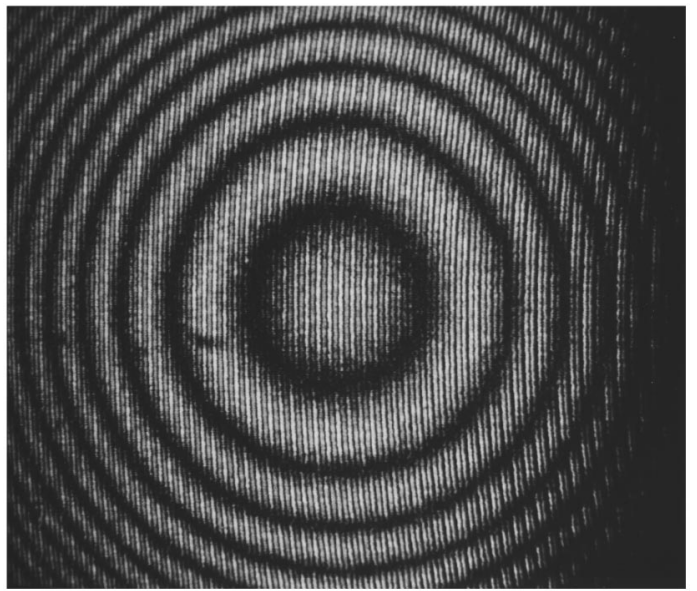

Fig. 5 Image displayed after high-pass filtering and rectification of the image shown in Figure 4. Now we see clear bright and dark bands, which are contours of equal height of the sphere's surface. The height difference between each dark (and each bright) contour is $0.082 \mathrm{~mm}$.

Instead of recording a flat plate as a reference image, we could record any other shape (such as the steel ball itself in some deformed state) and obtain fringes that directly give contours of equal height difference between the two shapes. We demonstrate this application in the next section.

The fringes seen in Figures 4 and 5 are contours of equal height difference between the spherical surface and the flat surface positioned parallel to the $x-y$ plane, and thus are contours of the spherical surface of equal height. We captured this image in a PC-based frame store (Data Translation 2151), and transferred the result to a matrix in Matlab (a matrix-based computation software package developed by The Math Works Inc.) for further analysis. Using this software package, we determined the $(x, y)$ coordinates of a number of points in the darkness center of the fringes and attributed a relative $z$ value to each point as the fringe plane distance times the order of the fringe. Then we fitted a best sphere through this set of three-dimensional data points. The diameter of the best-fitting sphere was $6.96 \mathrm{~mm}$ (with a fitting tolerance of $1 / 1000$ ), which deviates less than 3\% from the actual diameter of the steel ball.

Figure 6 shows the location of the fringe darkness centers along a horizontal section through the center of the sphere (indicated as circles), together with the best circle fitted through these points (indicated as a solid line). The aspect ratio of the figure has been strongly exaggerated so that the very small differences between the fitted best circle and the data points can be seen. At the right-hand side of the figure, an experimental data point is missing because the exact fringe location could not be determined due to the strong shading in that zone. The standard deviation of the difference between the data points and the $z$ values of the fitted circle is 


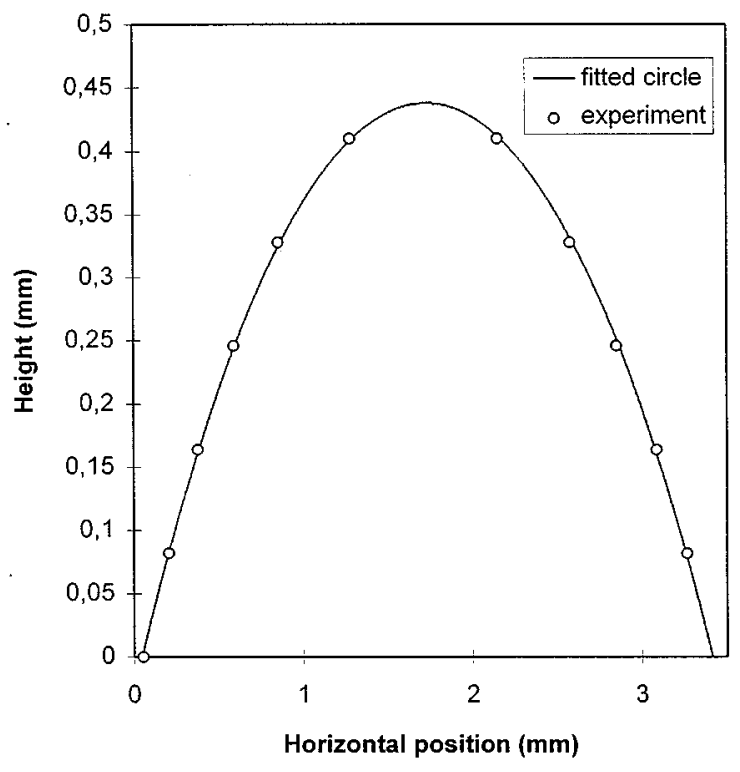

Fig. 6 Height of the sphere surface, measured by determining the order and the location of the fringe darkness centers along a horizontal line through the center of Figure 5. Data points are indicated with open circles. The solid line represents the best circle fitted through the data points. The aspect ratio of the figure is strongly exaggerated to reveal the very small deviations of the experimental data from the fitted curve.

$3 \mu \mathrm{m}$, which we can take as an estimate for the measuring precision of the setup. The maximum difference between the data points and the fitted circle is less than $5 \mu \mathrm{m}$. The total depth range of the measurement was $0.41 \mathrm{~mm}$, so the maximum deviation of the data points is $1.2 \%$ of the depth measuring range. Measurements on a sphere of $3 \mathrm{~mm}$ diameter yield values of similar accuracy and precision.

\section{APPLICATION EXAMPLE}

We developed this moiré interferometer initially for in vitro studies of the tympanic membrane. Its high sensitivity allows accurate measurement of the shape and the deformation of eardrums under small static pressures.

Figure 7 shows the shape of the moire interferogram obtained with our apparatus on an in vitro preparation of the tympanic membrane of a Mongolian gerbil. ${ }^{13,14}$ The middle ear has been removed so that the membrane can be seen from the middle ear side. The ear canal was filled with white Chinese ink to improve light reflectance. This approach is possible because the gerbil eardrum is nearly completely transparent. The fringes seen in Figure 7 represent contours of equal surface height, and demonstrate the conical shape of the tympanic membrane. They are the result of taking the difference between the grid image obtained on a flat plate and the grid image obtained on the eardrum.

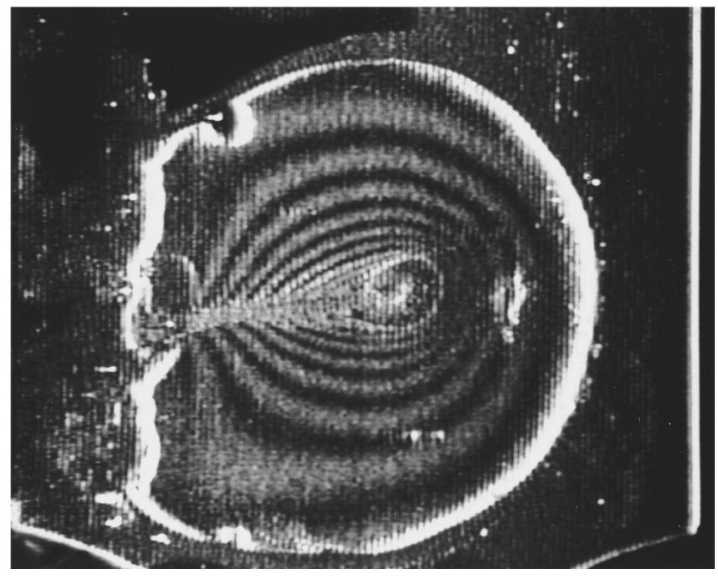

Fig. 7 Moiré interferogram obtained with our apparatus on a dissected gerbil tympanic membrane. To obtain the interferogram, the difference was taken between the grid line image on a flat plate and the grid line image on the membrane. The fringes represent contours of equal height, and demonstrate the conical shape of the membrane. The fringe plane distance, and hence the height difference per fringe order, is $0.082 \mathrm{~mm}$.

The fringe plane distance, and thus the height difference between successive fringe orders, was 0.082 $\mathrm{mm}$.

Static pressures can be applied to the eardrum through fluid ink in the ear canal. Figure 8 shows the moire interferogram of such a pressure-induced deformation. For this measurement, the difference was taken between the projected grid line image for the tympanic membrane in a resting state and the image obtained for the tympanic membrane under an overpressure of $0.2 \mathrm{kPa}$. Now the fringes represent contours of equal displacement. The fringe plane distance was the same as in the measurement presented in Figure 7, so the displacement increases with $0.082-\mathrm{mm}$ steps per contour order. The fringe

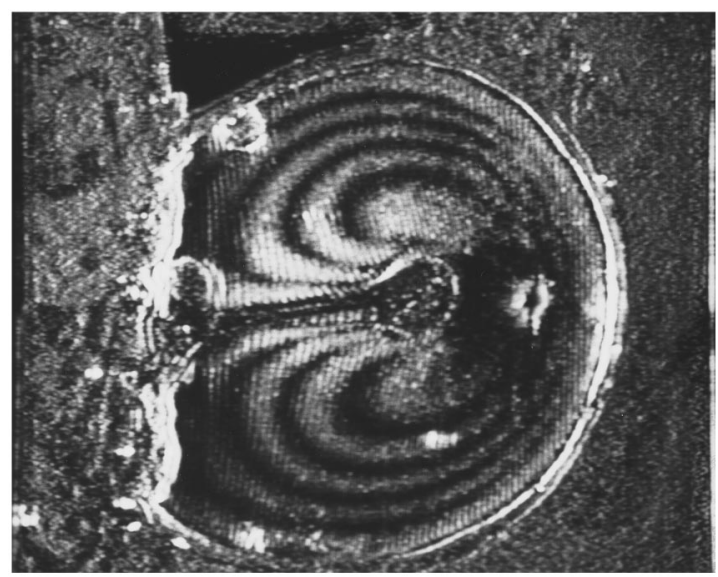

Fig. 8 Moire interferogram obtained with our apparatus on the same membrane as in Figure 6, but now with the difference taken between the grid line image on the membrane in its resting position and the grid line image on the membrane deformed by a static pressure of $2 \mathrm{kPa}$. The fringes now represent contours of equal displacement, again with a step of $0.082 \mathrm{~mm}$ per fringe order. 
pattern shows the characteristic two-lobed shape, which was also obtained by time-averaged holography measurements on eardrums vibrating at low frequencies. ${ }^{2}$

The spot on the right-hand side of the image is caused by specular reflections that saturate the imaging system. The shape measurement, shown in Figure 7, and the deformation measurement, represented in Figure 8, were obtained on the same membrane, in exactly the same position. Both figures are photographs of the display monitor, showing the result that is obtained in real time without any additional image enhancement.

\section{DISCUSSION}

In the practical setup, second-order effects of changing magnification and changing fringe plane distance with object depth limit the actual measuring precision. The measurements on the calibrated sphere, however, show that at a depth range of 0.4 $\mathrm{mm}$ the experimental data deviate less than $5 \mu \mathrm{m}$ from the actual surface height. This depth range suffices for the study of gerbil tympanic membranes, so shape and displacements can be measured with high accuracy. For objects having a larger depth range, absolute measuring accuracy will be less, but relative to the measuring depth, accuracy will remain quite constant. Owing to the use of finite apertures (rather than theoretical point apertures), the number of usable fringe planes is limited to about 25, so that the maximum depth measuring range is about $2 \mathrm{~mm}$. For objects of other sizes, the optical setup geometry can be altered so that the fringe plane distance, and hence the depth measuring range, increases.

We have performed shadow moiré measurements on the tympanic membrane of human cadaver temporal bones, ${ }^{1}$ but found it difficult to remove enough bone so that the tympanic membrane can be brought close enough to the grid. For studies on animal material such as cats or Mongolian gerbils, it is even more difficult to drill or cut away the bone, which partially covers the eardrum, because the bone is very thin and brittle and the tympanic membrane is extremely delicate. Using a projection setup overcomes these problems. In contrast to our work on shadow moire topography, we can now mount the dissected animal bulla in free space. This also allows the application of a stream of water vapor-saturated air to the membrane to avoid dehydration of the specimen.

For research in otology, a real-time optoelectronic moiré device offers other interesting possibilities. Because interferograms are obtained in real time, it is possible to obtain a large number of measurements in a short time, so one can study the response of the membrane to pressure with a large number of small pressure steps. A short measurement time is necessary because the dissected middle ear and the eardrum tend to dehydrate and to stiffen quickly, even when water vapor is applied. This setup also allows us to study the time course of a deformation such as viscoelasticinduced creep following a sudden pressure step.

A major advantage of the apparatus lies in the fact that both shape and deformation measurements can be performed in immediate succession, without altering the optical setup and without touching the specimen. The demonstration application showed how, by a simple reset of the frame store, the setup allows one to obtain both shape and displacement fringes on the object sitting in the same position. We use the shape measurement to determine the exact position of the tympanic membrane in the coordinate system. The border of the tympanic membrane (annulus) lies in a plane. While positioning the specimen in the setup, we observe the real-time shape of the moiré fringes and mount the specimen so that concentric fringes can be seen. We then know that the tympanic membrane annulus plane lies perfectly parallel with the $\mathrm{x}-\mathrm{y}$ plane.

By storing a new reference image, deformation can be followed starting from any reference state. In studies of viscoelastic behavior, for instance, the reference image can be stored immediately after applying the pressure step. Displacement fringes will then appear if the pressure step is followed by viscoelastic creep.

Because the apparatus uses standard video signals, it is also possible to perform image subtraction and processing on images that have been recorded on videotape. In our experiments we often use this postprocessing capability. During a measurement, the direct image of the camera is constantly recorded on a VHS video recorder. If it turns out afterward that more information is needed, the video recorder signal is fed to the input of the frame store, and any image on the tape can be chosen as the new reference image to study deformations with respect to that object shape.

\section{CONCLUSIONS}

We have described an optoelectronic moiré projection interferometer for real-time shape and deformation measurements. Both shape and displacement fringes are obtained without changing the optical setup, and with the object remaining in exactly the same position. The interferograms are obtained in real time, at a rate of 25 images per second. The device therefore allows us to follow deformations that evolve in time, or to obtain a large number of different interferograms in a short measuring time.

Using a fringe plane distance of $82 \mu \mathrm{m}$, a precision of better than $5 \mu \mathrm{m}$ was demonstrated in a measuring field 3.5 by 2.9 by $0.41 \mathrm{~mm}$. Measurements on a calibrated spherical surface show an absolute measuring accuracy of better than 3\%. At least 25 fringe orders can be clearly visualized, so 
the maximum depth measuring range is about $2 \mathrm{~mm}$.

The interferometer was especially designed for shape and deformation studies of the tympanic membrane. It allows the acquisition of valuable information on the mechanical behavior of the eardrum and the middle ear under static pressure, both in healthy and diseased ears. By changing the setup geometry, the image field can be adapted to study objects of varying sizes.

\section{REFERENCES}

1. S. Khanna and J. Tonndorf, "Tympanic membrane vibrations in cats studied by time-averaged holography," J. Acoust. Soc. Am. 51, 1904-1920 (1972).

2. J. Tonndorf and S. M. Khanna, "Tympanic membrane vibrations in human cadaver ears studied by time-averaged holography," J. Acoust. Soc. Am. 52 (4), 1221-1233 (1972).

3. O. J. Lokberg, K. Hogmoen, and T. Gundersen, "Vibration measurement of the human tympanic membrane in vivo," Acta Otolaryngol 89, 37-42 (1980).

4. G. von Bally, "Holography in otology," in Holography in Medicine and Biology, G. von Bally Ed., pp. 183-197, Springer-Verlag, Berlin (1979).

5. W. M. Decraemer, S. M. Khanna, and R. J. Funnell, "Interferometric measurement of the amplitude and phase of tympanic membrane vibrations in cat," Hear. Res. 38, 1-18 (1989).

6. W. M. Decraemer, S. M. Khanna, and W. R. J. Funnell, "A method for determining three-dimensional vibration in the ear," Hear. Res. 77, 19-37 (1994).

7. M. S. M. G. Vlaming, A. M. H. J. Aertsen, and W. J. M. Epping, "Directional hearing in the grass frog (Rana temporaria L.): mechanical vibrations of the tympanic membrane," Hear. Res. 14, 191-201 (1984).
8. N. G. Toremalm, "Laser Doppler scanning of the vibrating tympanic membrane," Acta Otolaryngol. (Stockh.) Suppl. 458, 52-55 (1988)

9. K. S. Konradsson, A. Ivarsson, and G. Bank, "Computerized laser Doppler interferometric scanning of the vibrating tympanic membrane," Scand. Audiol. 16, 159-166 (1987).

10. N. Stasche, H.-J. Foth, K. Hörmann, A. Baker, and C. Huthoff, "Middle ear transmission disorders-tympanic membrane vibration analysis by laser-Doppler-vibrometry," Acta Otolaryngol. (Stockh.) 114, 59-63 (1994).

11. J. J. J. Dirckx and W. F. Decraemer, "Shape and derived geometrical parameters of the adult, human tympanic membrane measured with a phase-shift moiré interferometer," Hear. Res. 51, 107-122 (1991)

12. J. J. J. Dirckx and W. F. Decraemer, "Human tympanic membrane deformation under static pressure," Hear. Res. 51, 93-106 (1991).

13. M. von Unge, W. F. Decraemer, D. Bagger-Sjöbäck, and J. J. Dirckx, "Displacement of the gerbil tympanic membrane under static pressure variations measured with a real-time differential moiré interferometer," Hear. Res. 70, 229-242 (1993).

14. M. von Unge, W. F. Decraemer, J. J. Dirckx, and D. BaggerSjöbäck, "Shape and displacement patterns of the gerbil tympanic membrane in experimental otitis media with effusion," Hear. Res. 82, 184-196 (1995).

15. D. M. Meadows, W. O. Johnson, and J. B. Allen, "Generation of surface contours by moiré patterns," Appl. Opt. 9 (4), 942 947 (1970).

16. H. Takasaki, "Moiré topography," Appl. Opt. 9 (6), 14671472 (1970).

17. J. Der Hovanesian and Y. Y. Hung, "Moiré contour-sum contour-difference and vibration analysis of arbitrary objects," Appl. Opt. 10 (12), 2734-2738 (1971).

18. M. Halioua M., R. S. Krishnamurthy, H. Liu, and F. P. Chiang, "Projection moiré with moving gratings for automated 3-D topography," Appl. Opt. 22 (6), 850-855 (1983). 